\title{
CONSTRUCTIONS BY RULER AND COMPASS, TOGETHER WITH A FIXED CONIC
}

\author{
SEUNGJIN BAEK, INSONG CHOE ${ }^{\otimes}$, YOONHO JUNG, \\ DONGWOOK LEE and JUNGGYO SEO
}

(Received 5 November 2012; accepted 19 December 2012; first published online 8 March 2013)

\begin{abstract}
It is well known to be impossible to trisect an arbitrary angle and duplicate an arbitrary cube by a ruler and a compass. On the other hand, it is known from ancient times that these constructions can be performed when the use of several conic curves is allowed. In this paper, we prove that any point constructible from conics can be constructed using a ruler and a compass, together with a single fixed nondegenerate conic different from a circle.
\end{abstract}

2010 Mathematics subject classification: primary 51M15; secondary 12F05.

Keywords and phrases: conic-constructible points, ruler and compass, conics, geometric construction.

\section{Introduction}

Trisecting an arbitrary angle and doubling the cube by ruler and compass are two of the famous problems posed by the Greeks which are known to be unsolvable. The first proof of the impossibility for the geometric constructions is attributed to Wantzel (1837). For the history of this subject, we refer the reader to [4, pp. 25-26].

It is also well known that the trisection and duplication are possible if the use of one or more conic sections in addition to ruler and compass is allowed [5]. The old constructions used hyperbolas and parabolas as conic sections. Recently, Hummel [3] and Gibbins and Smolinsky [2] independently found the constructions using ellipses.

Gibbins and Smolinsky, at the end of their paper, asked if one can reduce the number of types of ellipses for the constructions involved. More specifically, in the final remark of his paper, Hummel asked if all points constructible from ellipses are constructible using the same ellipse in addition to a ruler and a compass. The goal of this paper is to show that the answer is yes. More generally, we prove that every conic-constructible point can be obtained using a ruler and a compass, together with

This paper grew from an R\&E project 'A study on the numbers constructible from conic sections' under the supervision of the second named author. This project was supported by Seoul Science High School during March-December 2012.

(C) 2013 Australian Mathematical Publishing Association Inc. 0004-9727/2013 \$16.00 
a single fixed nondegenerate conic different from a circle (Theorem 3.2). It can be pointed out that our result resembles the Poncelet-Steiner theorem which states that any construction with a ruler and a compass can be accomplished by a ruler together with a fixed circle and its centre (see [1, Section 3.6]).

In the next section we examine the precise meaning of conic constructibility. In Section 3 we state the main result and give a proof.

\section{Conic-constructible points}

The point $(x, y)$ in the plane $\mathbb{R}^{2}$ is identified with a complex number $x+i y \in \mathbb{C}$. Starting from the initial set $P=P_{0}$ of points, carry out the following drawings.

(i) Given two points in $P$, draw a line through the two points using a ruler.

(ii) Given two points $z_{1}$ and $z_{2}$ in $P$, draw a circle centred at $z_{1}$ with radius $\left|z_{1}-z_{2}\right|$, using a compass.

Let $P_{1}$ be the set of points by adjoining all the intersections of lines and circles from the above drawings. Now, replacing $P_{0}$ by $P_{1}$ and running the above process, we get the set $P_{2}$. Repeating this inductively, we get a sequence of sets

$$
P=P_{0} \subset P_{1} \subset P_{2} \subset \cdots .
$$

A point is called constructible from $P$ if it is inside $P_{\infty}:=\bigcup_{i=0}^{\infty} P_{i}$. A point is called constructible if it is constructible from the initial set $\{0,1\}$ in $\mathbb{C}$. It is well known that a point is constructible if and only if it lies in a subfield $F$ of $\mathbb{C}$ which has a finite sequence of subfields starting from rational numbers:

$$
\mathbb{Q}=F_{0} \subset F_{1} \subset F_{2} \subset \cdots \subset F_{n}=F
$$

such that $\left[F_{i+1}: F_{i}\right]=2$ for each $i$.

Now in addition to the above drawings (i) and (ii), consider a third.

(iii) Draw all parabolas, ellipses, and hyperbolas having foci in $P$, directrix lines which are obtained from (i), and eccentricities equal to the length of a segment $\overline{z_{1} z_{2}}$ for some $z_{1}, z_{2} \in P$.

Let $P=Q_{0}$ and let $Q_{1}$ be the set of points by adjoining all the intersections of lines and circles and conics from the drawings (i)-(iii). Replacing $Q_{0}$ by $Q_{1}$ and running the same kind of process, we get $Q_{2}$. Again by induction, we get a sequence of sets

$$
P=Q_{0} \subset Q_{1} \subset Q_{2} \subset \cdots .
$$

A point is called conic-constructible from $P$ if it is inside $Q_{\infty}:=\bigcup_{i=0}^{\infty} Q_{i}$. A point is called conic-constructible if it is conic-constructible from the initial set $\{0,1\}$ in $\mathbb{C}$.

Note that for any $P$, both the set of constructible points derived from $P$ and the set of conic-constructible points derived from $P$ are subfields of $\mathbb{C}$, because processes (i) and (ii) are already enough to produce the complex numbers given by the operations ,,$+- \times, \div$. The $x$-coordinates of the constructible (or conic-constructible) points derived from $P$ again form a subfield of $\mathbb{R}$, and are called constructible (or conicconstructible) numbers derived from $P$. Videla found a useful criterion. 
Proposition 2.1 [5, Theorem 1, 2].

(1) The set of conic-constructible points forms the smallest subfield of $\mathbb{C}$ containing 0,1 , and $i$ which is closed under conjugation, square roots and cube roots.

(2) A point in $\mathbb{C}$ is conic-constructible if and only if it is contained in a subfield of $\mathbb{C}$ of the form $\mathbb{Q}\left(\alpha_{1}, \alpha_{2}, \ldots, \alpha_{n}\right)$, where $\alpha_{1}^{k_{1}} \in \mathbb{Q}$ and $\alpha_{i}^{k_{i}} \in \mathbb{Q}\left(\alpha_{1}, \ldots, \alpha_{i-1}\right)$ for $2 \leq i \leq n$, where $k_{i} \in\{2,3\}$ for each $i=1,2, \ldots, n$.

The point of Proposition 2.1(2) is that the square and cube roots of a given complex number are precisely the constructions required to obtain all the conic-constructible points. In other words, providing the constructions for real cube roots and trisections of angles is sufficient to get all conic-constructible points.

Let $K$ be a subfield of $\mathbb{R}$ such that every positive number $x \in K$ has a square root in $K$. A line passing through two points of $K^{2}$ is called a line in $K$. A circle is called a circle in $K$ if its centre is in $K^{2}$ and it passes through a point in $K^{2}$. Similarly, a conic (parabola, ellipse, or hyperbola) is called a conic in $K$ if its foci are in $K^{2}$, its directrix line is in $K$, and its eccentricity is in $K$. Recall the following useful fact.

Proposition 2.2. Let E be a nondegenerate conic (different from a circle), defined by the equation $a x^{2}+b x y+c y^{2}+d x+e y+f=0$. Then $E$ is in $K$ if and only if the coefficients $a, b, c, d, e, f$ are in $K$ (after suitable rescaling).

Proof. The proof for the ellipse case is given in [2, Proposition 3] and the argument can be modified to work also for parabolas and hyperbolas.

\section{Result}

We introduce two more notions: $C$-constructible points and $e$-constructible points. Let $C$ be a nondegenerate conic in the field of constructible numbers. For the initial set $P \subset \mathbb{C}$, a point is $C$-constructible from $P$ if it is obtained by the same process as conic-constructible points except that the conics in step (iii) are confined to the fixed one, $C$.

We call an ellipse or a hyperbola of eccentricity $e>0$ 'regular' if it is given by the equation

$$
\left(1-e^{2}\right)(x-a)^{2}+(y-b)^{2}=\lambda^{2}
$$

for some $a, b, \lambda \in \mathbb{R}$. Also, we call a parabola regular if it is of the form

$$
x=\lambda(y-a)^{2}+b
$$

for some $a, b, \lambda \in \mathbb{R}$.

Let $e>0$ be a constructible number. A point is e-constructible from $P$ if it is obtained by the same process as conic-constructible points except that:

(1) the conics in step (iii) are confined to the regular ones of eccentricity $e$; and

(2) the intersections of two conics, neither of which is a circle, are not adjoined. 
In other words, (1) a conic is drawn only when it is regular with eccentricity $e$, and (2) for any drawn conic different from a circle, only the intersections with lines and circles are counted for the next stage.

As before, when $P=\{0,1\}$, we say $C$-constructible and $e$-constructible respectively, for short. By definition, if $C$ is a regular conic with eccentricity $e$, then

$$
C \text {-constructible } \Rightarrow e \text {-constructible } \Rightarrow \text { conic-constructible. }
$$

LEMMA 3.1. Let $e>0$ be any constructible number. Every conic-constructible point is e-constructible.

Proof. Let $z \in \mathbb{C}$ be a conic-constructible point. By Proposition 2.1, $z \in$ $\mathbb{Q}\left(\alpha_{1}, \alpha_{2}, \ldots, \alpha_{n}\right)$, where either $\alpha_{i}^{2}$ or $\alpha_{i}^{3}$ is contained in $\mathbb{Q}\left(\alpha_{1}, \ldots, \alpha_{i-1}\right)$ for each $i$. We will show that $z$ is $e$-constructible by induction on $n$. Suppose that all the points in $H:=\mathbb{Q}\left(\alpha_{1}, \alpha_{2}, \ldots, \alpha_{n-1}\right)$ are $e$-constructible. If $\alpha_{n}^{2} \in H$, then $\alpha_{n}$ is constructible from $H$.

Now suppose that $\alpha_{n}^{3}=r e^{i \theta} \in H$. Let $q=\cos \theta$ and let $K$ be the field of constructible numbers derived from 0,1 and $r, q$.

First consider the intersection of the following circle and conic:

$$
x^{2}+y^{2}-r x-y=0 \quad \text { and } \quad\left(1-e^{2}\right) x^{2}+y^{2}-r x-\left(1-e^{2}\right) y=0 .
$$

By Proposition 2.2, these are a circle and a conic in $K$. From these two equations, we get $x^{4}-r x=0$. Hence the $x$-coordinate of the intersection points other than the origin corresponds to the cube root of $r$.

Next for $q=\cos \theta$, by triple-angle formula, $\cos (\theta / 3)$ is a real solution to the equation

$$
4 x^{3}-3 x-q=0
$$

To get this equation, we intersect the following circle and conic:

$$
x^{2}+y^{2}-\frac{q}{4} x-\frac{7}{4} y=0 \quad \text { and } \quad\left(1-e^{2}\right) x^{2}+y^{2}-\frac{q}{4} x-\left(\frac{7}{4}-e^{2}\right) y=0 .
$$

Again, these are a circle and a conic in $K$. Note that the conics in the above are regular of eccentricity $e$. From these intersections, we construct the numbers $\sqrt[3]{r}$ and $\cos (\theta / 3)$, and eventually the point $\alpha_{n}$. Thus so far we have shown that $\alpha_{n}$ is an $e$ constructible point derived from 0,1 , and $r, q$. Since we assumed that all the points in $H$ are $e$-constructible and $\alpha_{n}^{3}=r e^{i \theta} \in H, r$ and $q=\cos \theta$ are also $e$-constructible. Therefore, $\alpha_{n}$ is $e$-constructible and thus all the points in $\mathbb{Q}\left(\alpha_{1}, \alpha_{2}, \ldots, \alpha_{n}\right)$ are $e$-constructible.

Now comes our main result: every conic-constructible point can be constructed by using a single fixed conic in addition to a ruler and a compass. 
THEOREM 3.2. Let $C$ be any nondegenerate conic, different from a circle, in the field of constructible numbers. Then every conic-constructible point is $C$-constructible.

Proof. Let $e>0$ be any constructible number. By Lemma 3.1, it suffices to prove that every $e$-constructible point is $C$-constructible. We first introduce a hierarchy on the $e$-constructible points starting from the field of constructible points $\mathbb{F}_{0}$ :

$$
\mathbb{F}_{0} \subset \mathbb{F}_{1} \subset \mathbb{F}_{2} \subset \cdots
$$

Draw all the regular conics in $\mathbb{F}_{0}$ of eccentricity $e$. Let $Q_{1}^{e}$ be the set of points by adjoining to $\mathbb{F}_{0}$ all the intersections of lines and circles with any of the drawn regular conics. Let $\mathbb{F}_{1}$ be the field of constructible points derived from $Q_{1}^{e}$.

Inductively, for each $k \geq 0$, let $Q_{k+1}^{e}$ be the set of points by adjoining to $\mathbb{F}_{k}$ all the intersections of lines and circles with any of the drawn regular conics in $\mathbb{F}_{k}$ of eccentricity $e$. And define $\mathbb{F}_{k+1}$ as the field of constructible points derived from $Q_{k+1}^{e}$. Then the set of $e$-constructible points coincides with $\bigcup_{k=0}^{\infty} \mathbb{F}_{k}$.

Now we prove that every $e$-constructible point is $C$-constructible by induction on $k$. Certainly the constructible points $\mathbb{F}_{0}$ are $C$-constructible. Now assume that all the points in $\mathbb{F}_{k}$ are $C$-constructible. Take any point $z \in \mathbb{F}_{k+1} \backslash \mathbb{F}_{k}$ which is obtained as an intersection of a circle $R_{k}$ in $\mathbb{F}_{k}$ and a regular conic $C_{k}$ in $\mathbb{F}_{k}$ of eccentricity $e$.

We may assume that $C$ is regular. Indeed, we may rotate $C$ by angle $\theta$ where $\cos \theta$ and $\sin \theta$ are constructible, to send it to a regular conic, because $C$ is in the field of constructible numbers. From now on, we assume that $C$ is regular.

Since both $C$ and $C_{k}$ are in $\mathbb{F}_{k}$ of the same eccentricity, $C_{k}$ can be obtained from $C$ by a magnification by a factor $\lambda>0$ and a translation in $a+b i$, where $a, b$ and $\lambda$ are in $\mathbb{F}_{k}$. Therefore, the intersection point $z \in R_{k} \cap C_{k}$ can be obtained as follows.

(a) Translate the circle $R_{k}$ by a point in $\mathbb{F}_{k}$ and magnify by a factor in $\mathbb{F}_{k}$ to get $R_{k}^{\prime}$.

(b) Intersect $R_{k}^{\prime}$ with $C$ to get the corresponding intersection point $z^{\prime}$.

(c) Reverse the process (a) to get $z$ from $z^{\prime}$.

Since we assumed that all of the points in $\mathbb{F}_{k}$ are $C$-constructible, the intersection $z^{\prime} \in R_{k}^{\prime} \cap C$ is a $C$-constructible point, because $R_{k}^{\prime}$ is a circle in $\mathbb{F}_{k}$. We conclude that $z$ is a $C$-constructible point.

Since every point in $\mathbb{F}_{k+1}$ is constructible from $\mathbb{F}_{k}$ and the intersection points $z \in R_{k} \cap C_{k}$ obtained as above, it is $C$-constructible. This completes the proof.

\section{References}

[1] H. Eves, College Geometry (Johns and Bartlett, Boston, 1995).

[2] A. Gibbins and L. Smolinsky, 'Geometric constructions with ellipses', Math. Intelligencer 31(1) (2009), 57-62.

[3] P. Hummel, 'Solid constructions using conics', Pi Mu Epsilon J. 11(8) (2003), 429-435.

[4] J. Stillwell, Mathematics and its History, 2nd edn. Undergraduate Texts in Mathematics (Springer, New York, 2002).

[5] C. R. Videla, 'On points constructible from conics', Math. Intelligencer 19(2) (1997), 53-57. 
SEUNGJIN BAEK, Seoul Science High School, Uamgil 63 (Hyewha-dong 1-1), Jongro-ku, Seoul 110-530, Korea

e-mail: bjh7790@naver.com

INSONG CHOE, Department of Mathematics, Konkuk University, 1 Hwayang-dong, Gwangjin-Gu, Seoul 143-701, Korea

e-mail: ischoe@konkuk.ac.kr

YOONHO JUNG, Seoul Science High School, Uamgil 63 (Hyewha-dong 1-1), Jongro-ku, Seoul 110-530, Korea

e-mail: yoonhoim@nate.com

DONGWOOK LEE, Seoul Science High School, Uamgil 63 (Hyewha-dong 1-1), Jongro-ku, Seoul 110-530, Korea

e-mail: dwleeid@naver.com

JUNGGYO SEO, Seoul Science High School, Uamgil 63 (Hyewha-dong 1-1), Jongro-ku, Seoul 110-530, Korea

e-mail: sjgceo@naver.com 